\title{
All-trans retinoic acid-incorporated nanoparticles of deoxycholic acid-conjugated dextran for treatment of CT26 colorectal carcinoma cells
}

This article was published in the following Dove Press journal:

International Journal of Nanomedicine

29 January 2013

Number of times this article has been viewed

\author{
Young II Jeongl,* \\ Kyu Don Chung $2, *$ \\ Da Hye Kim ${ }^{3}$ \\ Yoon Hyuk Kim ${ }^{4}$ \\ Yeon Soo Lee $^{5}$ \\ $\mathrm{Ki}$ Choon $\mathrm{Choi}^{3}$ \\ 'Cancer Center, Chonnam National \\ University Hwasun Hospital, Hwasun, \\ South Korea; ${ }^{2}$ Department of \\ Anesthesiology and Pain Medicine, \\ Catholic University, Seoul, \\ South Korea; ${ }^{3}$ Grassland and Forage \\ Research Center, Rural Development \\ Administration, Cheonan, \\ South Korea; ${ }^{4}$ Department of \\ Mechanical Engineering, Kyung Hee \\ University, Seoul, South Korea; \\ ${ }^{5}$ Department of Biomedical \\ Engineering, Catholic University \\ of Daegu, Daegu, South Korea \\ *These authors equally contributed \\ to this work
}

Purpose: All-trans retinoic acid (RA)-incorporated nanoparticles were prepared using deoxycholic acid-conjugated dextran (DexDA). Anticancer activity of RA-incorporated DexDA nanoparticles were tested in vitro and in vivo.

Methods: RA-incorporated nanoparticles were prepared by dialysis. Antiproliferative and anti-invasive potential of RA-incorporated nanoparticles were studied using CT26 colorectal carcinoma cells.

Results: RA-incorporated nanoparticles have small particle sizes of around 70-300 nm and spherical shapes. The higher drug-feeding ratio and higher substitution degree of deoxycholic acid in the conjugates resulted in higher drug contents, lower loading efficiency, and larger particle size. RA release rate became slower at higher drug contents and higher substitution degree of deoxycholic acid in the DexDA conjugates. The antiproliferation activity, anti-invasive activity, and matrix metalloproteinase 2 expression of RA-incorporated nanoparticles against CT26 cells in vitro was similar to RA. However, RA-incorporated nanoparticles had superior antimetastatic activity in an animal pulmonary metastatic model of CT26 cells compared to RA itself.

Conclusion: RA-incorporated nanoparticles showed similar anticancer activity in vitro and superior antimetastatic activity in vivo in a pulmonary metastatic model of CT26 cells. We suggest that RA-incorporated nanoparticles are promising vehicles for efficient delivery of RA.

Keywords: nanoparticle, dextran, deoxycholic acid, retinoic acid, cancer cell invasion

\section{Introduction}

Self-organized nanoaggregates, such as nanoparticles, polymeric micelles, and selfassembled vehicles, have been extensively investigated in the field of biomedicine and drug-delivery system. ${ }^{1-4}$ In particular, since nanoaggregates have small particle sizes of less than $1000 \mathrm{~nm}$, they are frequently considered for targeted delivery of anticancer drugs to the site of action. ${ }^{1-7}$ They have such advantages as solubilization of hydrophobic drugs, reduction of total dose of drug administered, prevention of undesirable side effects, and non-necessity of surgical removal after use. ${ }^{1-7}$ In particular, nanoparticles based on amphiphilic macromolecules have an inherent structure such that the hydrophobic domain of amphiphilic macromolecules composes the inner core of the nanoparticles for drug incorporation and the hydrophilic domain composes the surrounding region of the nanoparticles. These intrinsic structures enable nanoparticles to avoid attack of reticuloendothelial system and increase of blood circulation time..$^{5-7}$ 
All-trans retinoic acid (RA) has been reported to be effective in the treatment of various tumors, such as human malignant glioma, breast cancer, head and neck cancer, ovarian adenocarcinoma, and acute promyelocytic leukemia. ${ }^{8-15}$ In addition, RA is known to have antiproliferative, antimigration, and anti-invasion efficacy against various tumors. ${ }^{13}$ However, drawbacks such as retinoid acute resistance, hypertriglyceridemia, mucocutaneous dryness, headache, cancer relapse after a brief remission, drug resistance, and low plasma RA concentration limit its clinical application. ${ }^{15-17}$ Furthermore, its poor aqueous solubility $(0.21 \mathrm{mM}$ in physiological solution, $\mathrm{pH} 7.3$ ) is also problematic for clinical application. ${ }^{18}$ To overcome these problems, various kinds of vehicles for delivery of RA have been developed in the form of microspheres, liposomes, lipid nanoparticles, and polymeric micelles. ${ }^{19-23}$

In this study, we prepared RA-incorporated nanoparticles using deoxycholic acid (DA)-conjugated dextran (DexDA) conjugates. Drug-release and physicochemical properties of RA-incorporated nanoparticles of DexDA were studied in vitro. Furthermore, their anticancer activities were investigated against CT26 colorectal carcinoma cells in vitro.

\section{Materials and methods}

\section{Materials}

Dextran from Leuconostoc mesenteroides (average molecular weights 38,900), RA, deoxycholic acid (DA), thiazolyl blue tetrazolium bromide (MTT), $\mathrm{N}, \mathrm{N}^{\prime}$ - Dicyclohexylcarbodiimide (DCC), and 4-(N,N-dimethylamino)pyridine (DMAP) were purchased from Sigma-Aldrich (St Louis, MO, USA). The dialysis membranes (molecular weight cutoff $8000 \mathrm{~g} / \mathrm{mol}$ ) were purchased from Spectra Laboratories (Rancho Dominguez, CA, USA). Dichloromethane and dimethyl sulfoxide (DMSO) used were of extra-pure grade.

\section{Synthesis of DexDA conjugates}

DexDA conjugates were synthesized as previously reported. ${ }^{24}$ DA was conjugated to the hydroxyl group of glucose units of dextran by carbodiimide chemistry. Dextran and DMAP were dissolved in anhydrous DMSO. DA and 1.5 equivalent DCC were dissolved in DMSO and stirred for 6 hours to activate the carboxyl group of DA. DA/DCC solution was added to the dextran/DMAP solution. This solution was kept to react for 2 days. After that, this solution was filtered to remove the byproducts and dialyzed against distilled water for at least 3 days, with exchange of water at intervals of 2-3 hours.
The resultant solution was freeze-dried for 2 days and its solid in white obtained. This solid was purified again by precipitation into dichloromethane for three times to remove unreacted elements. The resulting product was dried in a vacuum oven for 2 days.

The substitution degree (DS) of DA to dextran was evaluated from the ${ }^{1} \mathrm{H}$ nuclear magnetic resonance (NMR) spectra and expressed as mol\% versus 100 glucose units.

\section{'H nuclear magnetic resonance spectroscopy measurement}

Characterization of DexDA conjugates was performed in DMSO or $\mathrm{D}_{2} \mathrm{O}$ using $400 \mathrm{MHz}{ }^{1} \mathrm{H}$ NMR spectroscopy (Varian 400 MHz NMR; Agilent Technologies, Santa Clara, CA, USA).

\section{Morphological observation of nanoparticles}

The morphology of the nanoparticles was observed using a transmission electron microscope (TEM) (JEM-2000 FX II; JEOL, Tokyo, Japan). One drop of nanoparticle solution containing phosphotungstic acid $(0.05 \%[\mathrm{w} / \mathrm{w}])$ was placed onto a carbon film coated on a copper grid for TEM. Observation was done at $80 \mathrm{kV}$.

\section{Particle-size measurement}

Particle size and zeta potential of nanoparticles were measured with dynamic laser scattering (DLS-7000; Otsuka Electronics, Osaka, Japan). A sample solution obtained right after the dialysis procedure was used for particle size (concentration: $0.1 \mathrm{wt} \%$ ).

\section{Preparation of RA-incorporated DexDA nanoparticles}

The RA-incorporated nanoparticles were prepared as follows: each $80-90 \mathrm{mg}$ of DexDA dissolved in $8 \mathrm{~mL}$ of DMSO was mixed with 10-20 mg RA in DMSO for 1 hour. This solution was poured into $20 \mathrm{~mL}$ of deionized water for 10 minutes to form RA-incorporated nanoparticles. DMSO and free drug were removed by dialysis against distilled water for at least 1 day. After that, the resulting solution was used for analysis or lyophilized.

For evaluation of drug content and loading efficiency, $5 \mathrm{mg}$ of RA-incorporated nanoparticles of DexDA were dissolved in $10 \mathrm{~mL}$ of DMSO and diluted 100 times. RA concentration was measured using a UV spectrophotometer (1201; Shimadzu, Kyoto, Japan) at 365 nm. Empty nanoparticles of DexDA were used as a blank test. 


$$
\begin{aligned}
& \text { Drug } \\
& \text { contents }
\end{aligned}=\frac{\text { Amount of RA in the nanoparticles }}{\text { Weight of nanoparticles }} \times 100
$$

$\begin{aligned} & \text { Loading } \\ & \text { efficiency }\end{aligned}=\frac{\text { Residual amount of RA in the nanoparticles }}{\text { Feeding amount of RA }} \times 100$

\section{Drug-release study in vitro}

The release experiment was carried out in vitro as follows: $5 \mathrm{mg}$ of RA-incorporated nanoparticles were distributed into $10 \mathrm{~mL}$ of phosphate-buffered saline (PBS; 0.1 M, pH 7.4), and this solution was introduced into dialysis membrane. After that, dialysis membrane was placed in a $200 \mathrm{~mL}$ bottle with $90 \mathrm{~mL}$ of PBS. This bottle was introduced in a shaking incubator with stirring speed of $100 \mathrm{rpm}$ at $37^{\circ} \mathrm{C}$. At specific time intervals, the media were taken for analysis of drug concentration. After that, the whole media was replaced with fresh PBS to prevent drug saturation. The concentration of the RA released into PBS was evaluated using the UV spectrophotometer at $365 \mathrm{~nm}$.

\section{Cell culture}

RAW264.7 mouse macrophage cells and CT26 colorectal carcinoma cells were purchased from the Korean Cell Line Bank (Seoul, South Korea).

\section{In vitro cell cytotoxicity}

For measurement of intrinsic cytotoxicity of empty nanoparticles of DexDA conjugates, RAW264.7 cells were employed. A total of $3 \times 10^{4}$ RAW264.7 cells seeded in 96-well plates with $100 \mu \mathrm{L}$ volume of media were exposed to empty nanoparticles. Empty nanoparticles of DexDA were reconstituted into fetal bovine serum (FBS) free Dulbecco's modified Eagle's medium and added to 96 wells. All treatment was incubated for 2 days. The viability of cells was evaluated by MTT assay. Briefly, $30 \mu \mathrm{L}$ of MTT $(5 \mathrm{mg} / \mathrm{mL})$ was added to 96 -well plates and incubated for 4 hours at $5 \%$ $\mathrm{CO}_{2}$ at $37^{\circ} \mathrm{C}$. The formazan crystals were then dissolved with DMSO and the absorbance $(560 \mathrm{~nm}$ test $/ 630 \mathrm{~nm}$ reference) was determined using an automated computerlinked microplate reader (Molecular Devices, Sunnyvale, CA, USA). Each measurement of the drug concentration was obtained as the mean value of eight wells. The amount of formazan present is proportional to the number of viable cells, as only living cells will reduce MTT to blue formazan. The results were expressed as a percentage of the absorbance present in the drug-treated cells compared to that in the control cells.
Proliferation inhibition of RA and RA-incorporated nanoparticles against CT26 mouse-colon carcinoma cells was evaluated in vitro. CT26 cells were cultured with Roswell Park Memorial Institute (RPMI) medium 1640 (10\% FBS), $5 \% \mathrm{CO}_{2}$ at $37^{\circ} \mathrm{C}$. A total of $1 \times 10^{4} \mathrm{CT} 26$ cells seeded in 96-well plates with $100 \mu \mathrm{L}$ volume of media were exposed to drug or nanoparticles. For RA itself, RA was dissolved in DMSO and diluted 100 times with RPMI 1640 $(+10 \% \mathrm{FBS})$ and added to 96 wells (final concentration of DMSO was $0.5 \% \mathrm{v} / \mathrm{v})$. For RA-incorporated nanoparticles, lyophilized nanoparticles were reconstituted into RPMI $1640(+10 \%$ FBS $)$ and added to 96 wells. For comparison, empty nanoparticles were also distributed in RPMI 1640 $(+10 \%$ FBS $)$ and then added to 96 wells. All treatment against tumor cells was incubated for 1 or 2 days. The growth inhibition of RA or nanoparticles was evaluated by MTT assay.

\section{Gelatin zymography and Matrigel invasion assay}

CT26 cells were grown to enrich in the $6 \mathrm{~cm}$ culture plate. After that, the medium was replaced by $1 \mathrm{~mL}$ of serum-free media with or without drug. After 24 hours, the conditioned media was harvested and ultracentrifuged to remove the cell debris for gelatin zymography assay. The centrifuged solution (total protein $30 \mu \mathrm{g}$ ) was mixed with the sample buffer (50 mM Tris-HCl, $2 \%$ sodium dodecyl sulfate, $0.1 \%$ bromophenol blue, $10 \%$ glycerol) and electrophoresed on $8 \%$ denaturing dodecyl sulfate polyacrylamide gels containing $2 \mathrm{mg} / \mathrm{mL}$ of gelatin (type A, Sigma-Aldrich). After that, gels were washed with $2.5 \%$ Triton X-100 and then incubated for 48 hours at $37^{\circ} \mathrm{C}$ in Tris- $\mathrm{HCl}$ buffer solution (pH 7.5, $10 \mathrm{mM} \mathrm{CaCl}_{2}$, and $200 \mathrm{mM} \mathrm{NaCl}$ ). The gels were stained with Coomassie Brilliant Blue R-250 (0.2\% Coomassie Brilliant Blue, $20 \%$ methanol, $10 \%$ acetic acid in $\mathrm{H}_{2} \mathrm{O}$ ) and destained (20\% methanol, $10 \%$ acetic acid in $\left.\mathrm{H}_{2} \mathrm{O}\right)$.

For Matrigel invasion assay, a Boyden chamber with filter inserts was employed. Prior to invasion assay, CT26 cells were exposed to serum-free media for the control, empty nanoparticles $(100 \mu \mathrm{g} / \mathrm{mL}), \mathrm{RA}$, and RA-incorporated nanoparticles. After 24 hours, cells were trypsinized and washed with PBS for use in invasion assay. Filter inserts in 24-well plates containing $8 \mu \mathrm{m}$ pores were coated with Matrigel. CT26 cells (30,000 cells/well) were suspended in serum-free medium and plated into the upper chamber of filter inserts, and then the lower chamber was filled with Dulbecco's modified Eagle's medium containing 10\% FBS. After 48 hours, non-invaded cells on the upper chamber 
were gently removed, and invaded cells were stained with the solution in a cell-invasion assay kit (ECM550; Chemicon International, Temecula, CA, USA) following counting with microscopic examination.

\section{In vivo CT26 pulmonary metastasis model}

The pulmonary metastasis of CT26 cells was prepared by a previously described method. ${ }^{25,26} \mathrm{CT} 26$ cells were administered into the tail vein of BALb/C mice $\left(1 \times 10^{5}\right.$ cells $/ 0.2 \mathrm{~mL}$ Hank's balanced salt solution). RA was dissolved in 5\% HCO-60 (Nicco Chemicals, Tokyo, Japan) solution, and RAincorporated nanoparticles were administered via the tail vein (RA dose $5 \mathrm{mg} / \mathrm{kg}$ ) at day 3 after tumor inoculation. After 2 weeks, mice were killed to extract lungs. The number of tumor nodules on the lung surface was counted and compared to control (PBS, 0.01 M, pH 7.4).

\section{Statistical analysis}

Results of animal experiments were expressed as average \pm standard deviation, and statistical significance was evaluated using Student's $t$-test. A $P$-value $<0.05$ was considered significant.

\section{Results}

\section{Characterization of RA-incorporated DexDA nanoparticles}

Since dextran itself is fully hydrophilic and not able to form nanoaggregates, DA as a hydrophobic moiety was conjugated to dextran, as previously reported. ${ }^{24}$ Chemical structure of DexDA conjugates and DS of DA was confirmed by ${ }^{1} \mathrm{H}$ NMR spectra (data not shown). Specific peaks of dextran appeared at 3.0-3.8 ppm and 4.5-5.0 ppm, while intrinsic peaks of DA appeared at 0.5-2.4 ppm and 3.0-4.5 ppm. The DS of DA was estimated by comparison of proton peak ratio between $0.5-2.0 \mathrm{ppm}$ of DA and $3.7 \mathrm{ppm}$ of dextran. DS value of DA was increased by increase of DA feed ratio (Table 1 ).
DexDA conjugates were used to incorporate RA and to form nanoparticles for antitumor drug delivery. To confirm nanoparticle formation, morphology of RA-incorporated DexDA-2 nanoparticles was observed using TEM, as shown in Figure 1. These have spherical shapes and small average particle size of less than $100 \mathrm{~nm}$. When the feeding amount of RA was increased, drug content increased but loading efficiency decreased. When higher DS of DA conjugation was used, drug content and loading efficiency increased. These results were due to the increased hydrophobic interaction between hydrophobic DA moiety in the DexDA conjugates and hydrophobic drug, as shown in Table 1. Furthermore, increased drug content induced larger particles, ie, particle size reached more than $300 \mathrm{~nm}$, as shown in Figure 2A. As shown in Figure 2B, the higher hydrophobic DA moiety resulted in increased particle size. These results might be due to the fact that higher hydrophobic moiety induced higher hydrophobic interaction and then resulted in higher drug content/particle size.

\section{Drug release from DexDA nanoparticles}

RA release from DexDA nanoparticles was studied in vitro. As shown in Figure 3A, the higher drug content resulted in the decreased release rate of drug. Furthermore, increased DS of DA in the DexDA conjugates led to a decreased drugrelease rate from the nanoparticles (Figure 3B). These results indicated that hydrophobic interaction might be increased between drug and polymer at higher drug loading and higher DS of DA in the DexDA conjugates. Furthermore, increased hydrophobic interaction frequently induces crystallization or aggregation of drug in the core of the nanoparticles. Then, crystallized drug in the nanoparticles may have reduced solubility against water and decreased release kinetics., 3,720

\section{Antitumor activity of RA-incorporated DexDA nanoparticles}

To test the intrinsic toxicity of empty nanoparticles of DexDA, RAW264.7 macrophage cells were employed. As shown in

Table I Characterization of RA-incorporated DexDA nanoparticles

\begin{tabular}{lllll}
\hline & $\begin{array}{l}\text { Substitution degree } \\
\text { of deoxycholic acid }\end{array}$ & $\begin{array}{l}\text { DexDA/ATRA weight } \\
\text { ratio }(\mathbf{m g} / \mathbf{m g})\end{array}$ & $\begin{array}{l}\text { Drug contents } \\
(\%, \mathbf{w} / \mathbf{w})\end{array}$ & $\begin{array}{l}\text { Loading efficiency } \\
\text { (\%, w/w) }\end{array}$ \\
\hline DexDA-I & 4.1 & $45 / 5$ & 7.1 & 68.8 \\
DexDA-2 & 8.7 & $50 / 0$ & - & - \\
& & $45 / 4$ & 8.2 & 80.4 \\
DexDA-3 & 15.6 & $40 / 10$ & 13.8 & 64.0 \\
\hline
\end{tabular}

Notes: ${ }^{a}$ Degree of substitution (DS) of deoxycholic acid was expressed based on number of deoxycholic acid versus 100 glucose unit; DS\% was calculated from proton nuclear magnetic resonance data.

Abbreviations: RA, all-trans retinoic acid; DexDA, deoxycholic acid-conjugated dextran. 

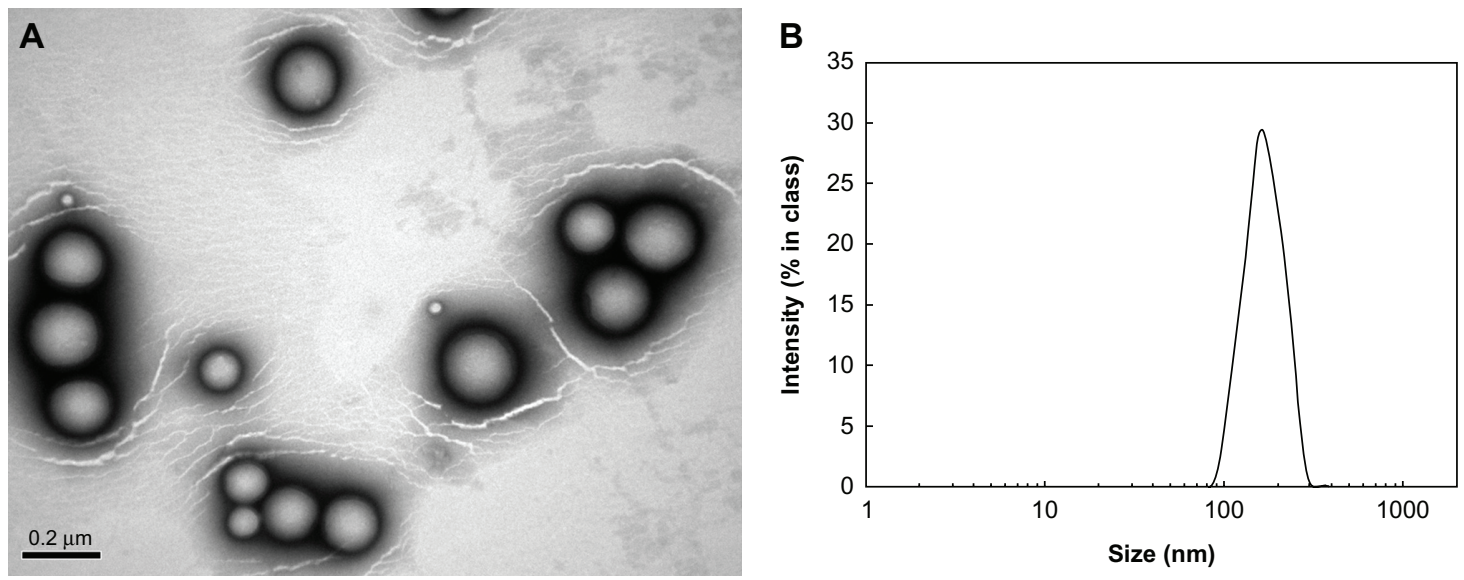

Figure I Typical particle-size distribution (A) and morphology (B) of RA-incorporated DexDA-2 nanoparticles.

Figure 4, DexDA nanoparticles in the absence of drug did not significantly affect the viability of cells, even though the viability of RAW264.7 cells was slightly affected by treatment of a higher concentration of DexDA conjugates. These results indicated that DexDA conjugates do not affect the viability of cells and were biocompatible with the cells.
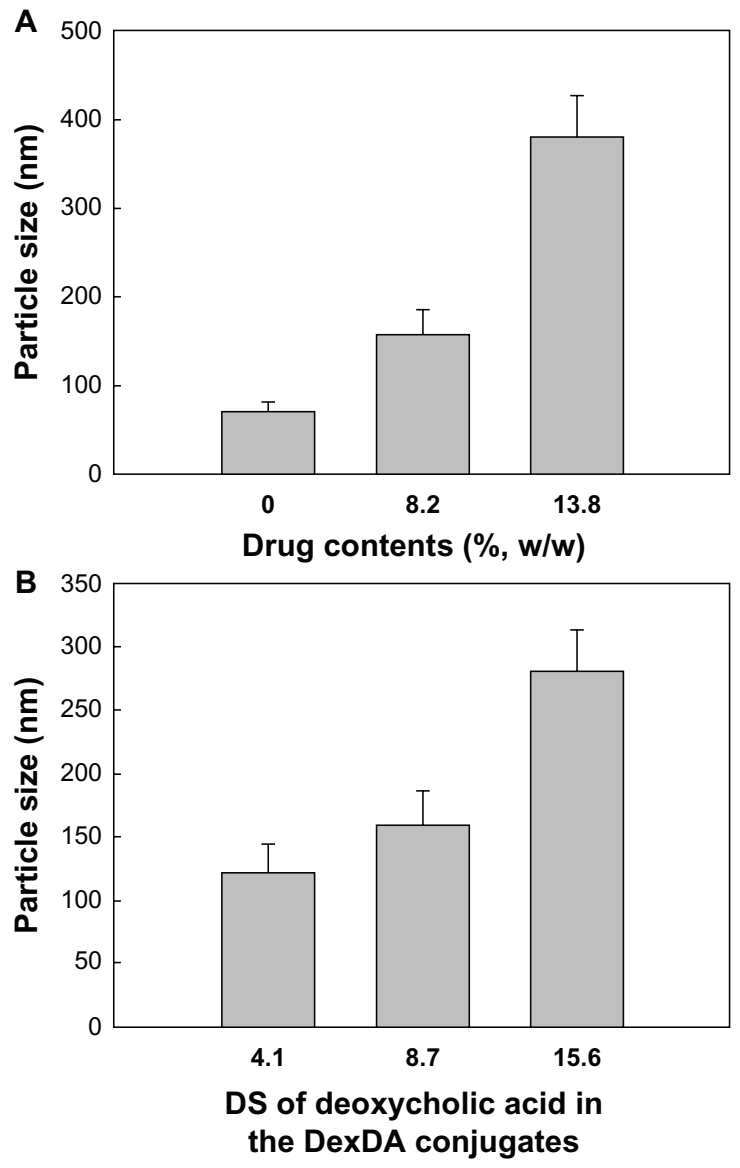

Figure 2 Particle-size changes according to the drug content in the nanoparticles (A) and substitution degree (DS) of deoxycholic acid in the deoxycholic acid-conjugated dextran (DexDA) conjugates $(\mathbf{B})$.
The antitumor effect of RA or nanoparticles was evaluated using CT26 colorectal carcinoma cells. The antiproliferation effect of RA or nanoparticles was assessed by MTT proliferation assay, as shown in Figure 5. The proliferation of tumor cells was dose-dependently inhibited by RA and RAincorporated DexDA nanoparticles, as shown in Figure 5.

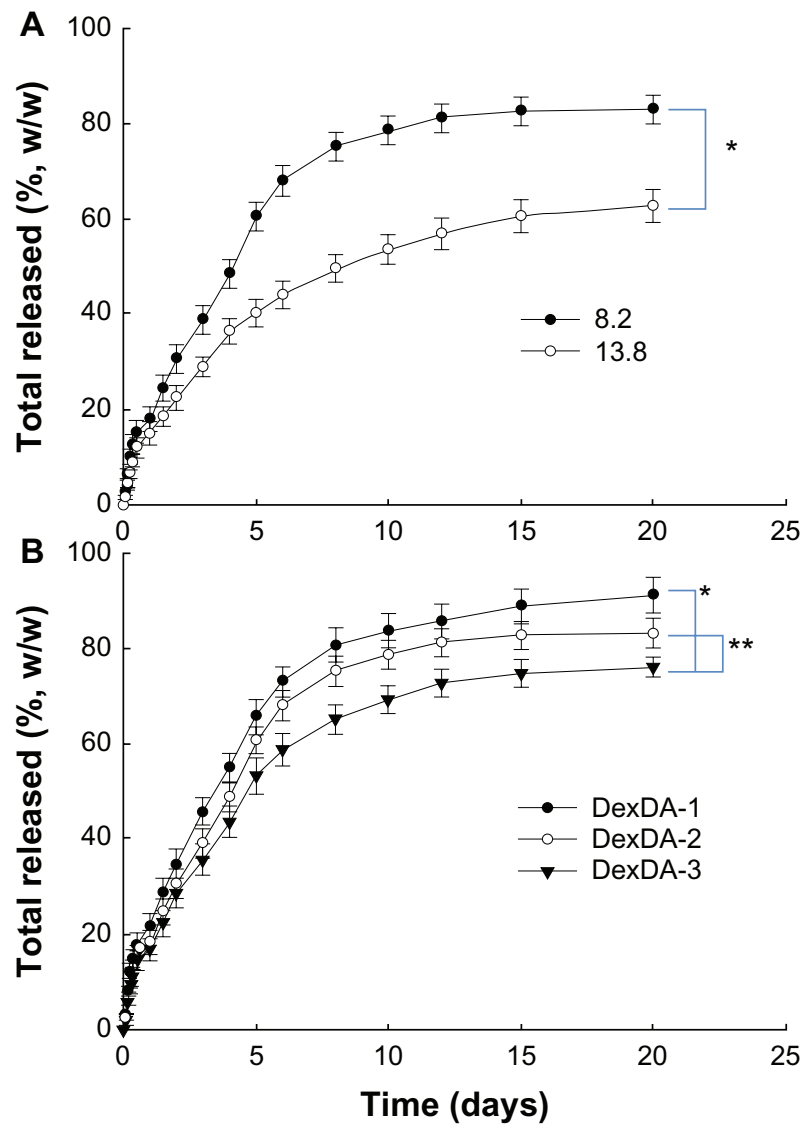

Figure 3 (A and B) RA release from DexDA nanoparticles. (A) The effect of drug content in the nanoparticles; (B) effect of substitution degree of deoxycholic acid in the conjugates.

Notes: $* P<0.001 ; * * p<0.01$. 


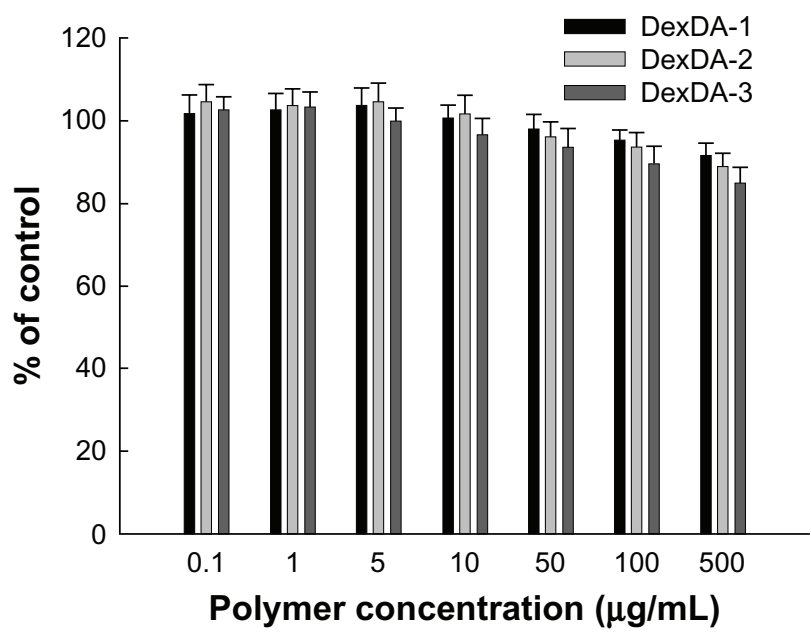

Figure 4 Intrinsic cytotoxicity of empty nanoparticles of deoxycholic acidconjugated dextran (DexDA) against RAW 264.7 mouse macrophage cells.

Notes: For intrinsic cytotoxicity of empty nanoparticles of DexDA conjugates, $3 \times 10^{4}$ RAW264.7 mouse macrophage cells were seeded in 96-well plates and exposed to empty DexDA nanoparticles. All treatment was incubated for 2 days. The viability of cells was evaluated by thiazolyl blue tetrazolium bromide (MTT) assay, as described in the Materials and methods section.

Since empty nanoparticles of DexDA-1, 2 and 3 did not significantly affect to the viability of normal cells (Figure 4) and tumor cells (data not shown), ${ }^{24}$ the antiproliferative effect of RA-incorporated nanoparticles against tumor cells was practically similar to RA itself.

\section{Anti-invasive activity of RA-incorporated DexDA nanoaggregates}

Anti-invasive or antimetastatic antitumor activity of RA or RA-incorporated nanoparticles against CT26 cells in vitro and pulmonary metastasis model in vivo were investigated, as shown in Figures 6 and 7.

To study the anti-invasive effect of RA-incorporated DexDA nanoparticles, Matrigel invasion assay was employed, as shown in Figure 6A. Prior to invasion assay and measurement of matrix metalloproteinase (MMP) expression, CT26 cells were exposed to various concentrations of empty nanoparticles, RA, and RA-incorporated DexDA-2 nanoparticles, as shown in Figure 6. CT26 cells treated with drug were seeded onto the Matrigel-coated upper chamber of the Boyden chamber. Invaded tumor cells across the Matrigel to the lower surfaces of the chamber were counted. As shown in Figure 6A, invasion of tumor cells was dose-dependently decreased by treatment of RA or RA-incorporated nanoparticles, while empty nanoparticles did not affect the invasive capability of tumor cells, indicating that RA-incorporated nanoparticles inhibited tumor cell invasion. The relative number of the invaded cells (graph in the left-middle

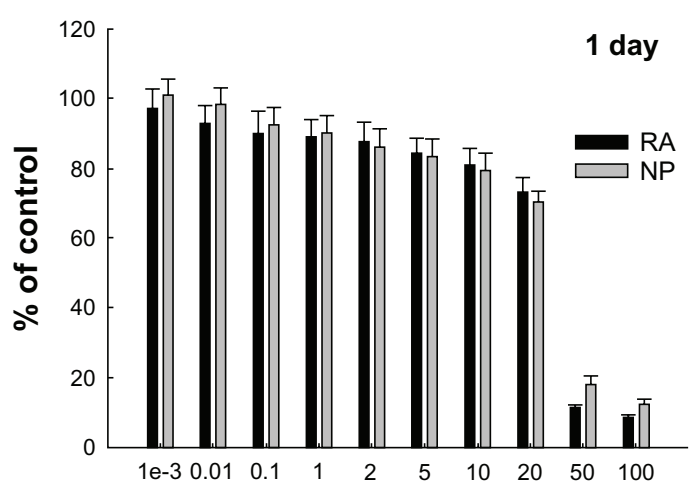

RA concentration (microgram $/ \mathrm{mL}$ )

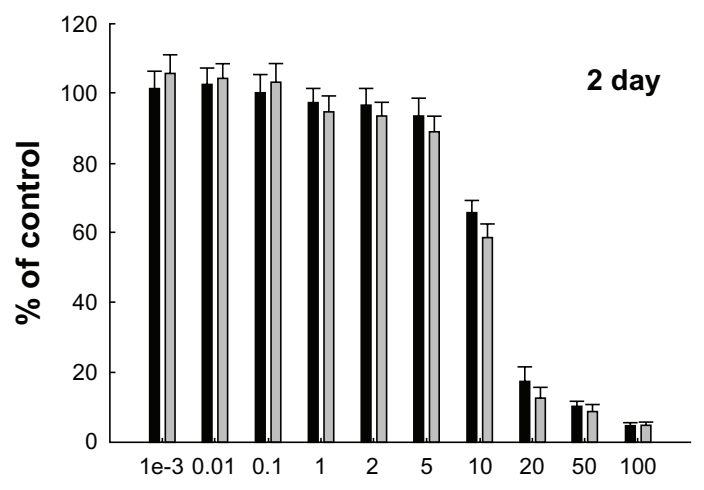

RA concentration $(\mu \mathrm{g} / \mathrm{mL})$

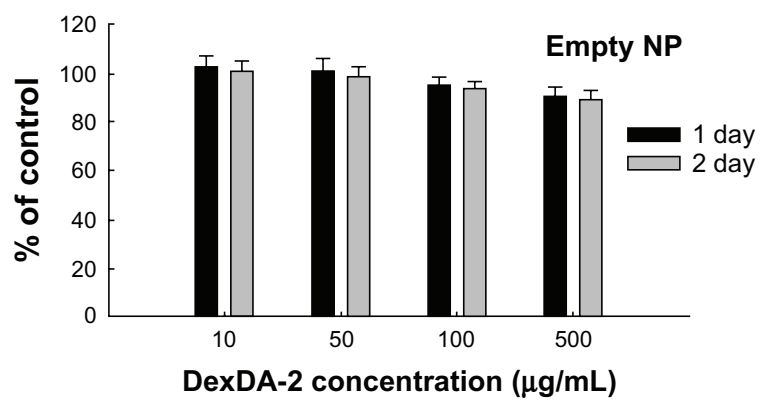

Figure 5 The effect of RA-incorporated DexDA-2 nanoparticles on the proliferation of CT26 tumor cells.

Abbreviations: RA, all-trans retinoic acid; DexDA, deoxycholic acid-conjugated dextran; NP, RA-incorporated nanoparticles of DexDA-2; empty NP, empty nanoparticles of DexDA-2.

position) was not significantly changed between RA itself and RA-incorporated nanoparticles. Furthermore, gelatin zymography was employed to study MMP-2 expression of CT26 cells, since MMP-2 is a key enzyme to degrade extracellular matrix and to promote invasion of tumor cells. As shown in Figure 6B, RA and its nanoparticles suppressed the MMP-2 expression of CT26 cells, clearly indicating that decrease of tumor cell invasion results from the suppression of MMP-2 expression of CT26 cells.

For in vivo pulmonary metastasis assay, CT26 cells were injected intravenously and the number of tumor nodules counted as an index of metastatic potential of tumor 
A
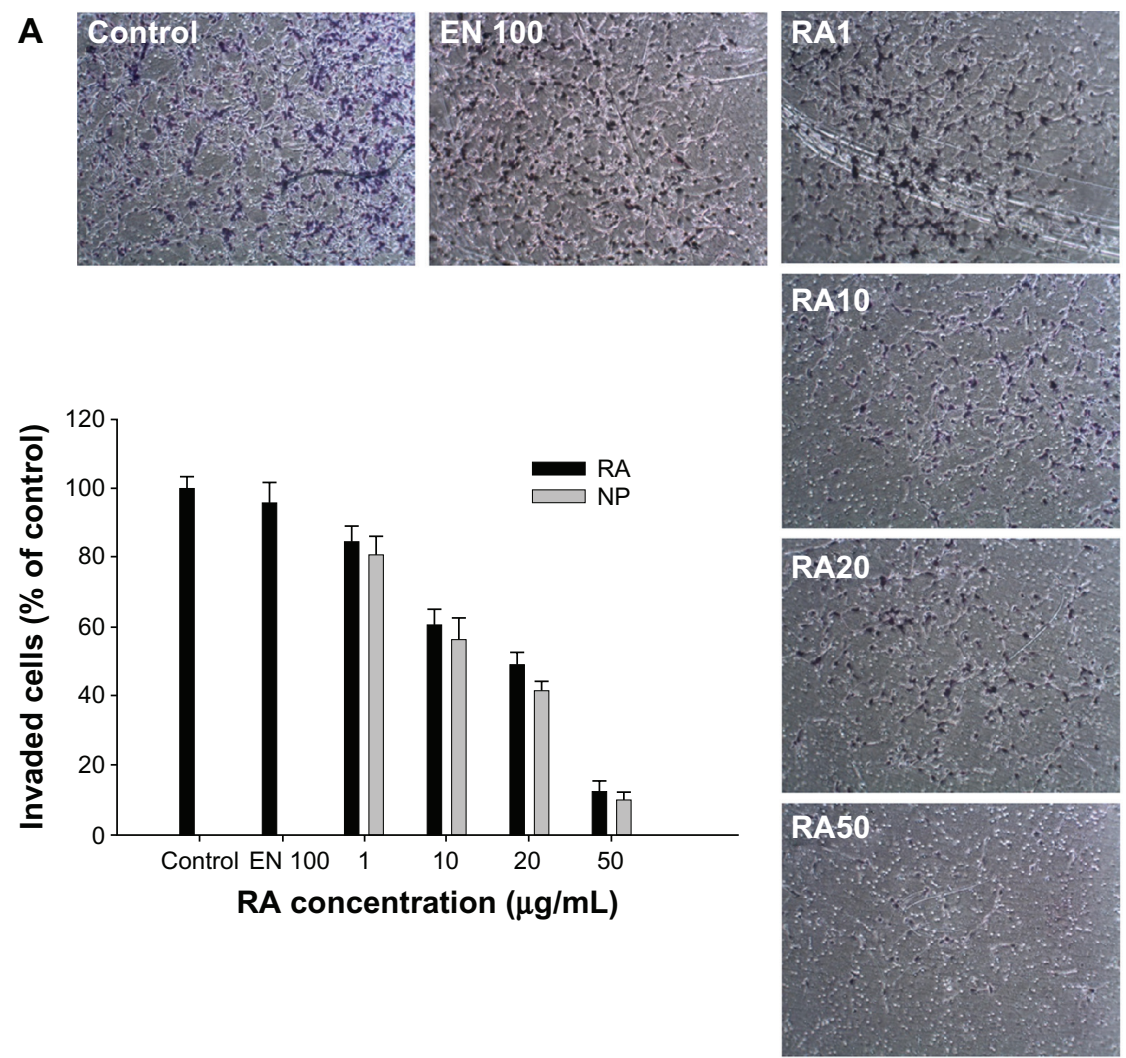
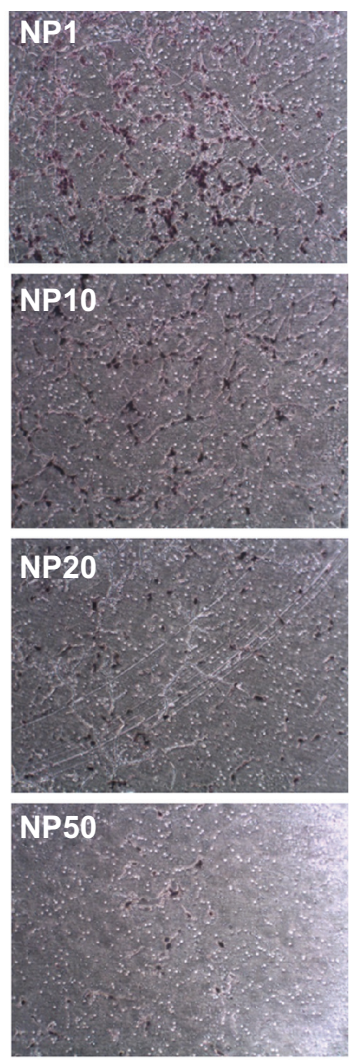

B

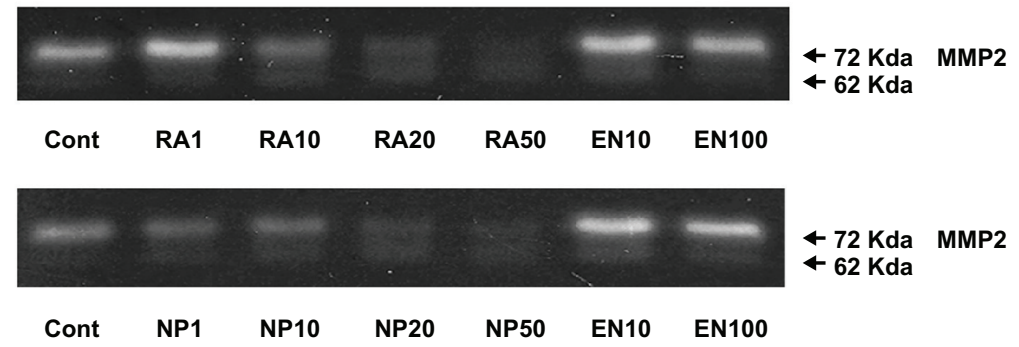

Figure 6 The effect of RA incorporated DexDA-2 nanoparticles on the invasion (A) and MMP-2 expression (B) of CT26 tumor cells.

Notes: Serum free media was treated for control. CT26 cells were exposed to empty nanoparticles, All-trans retinoic acid (RA), and RA-incorporated nanoparticles of DexDA-2 (NP) for 24h. After that, cells were trypsinized, washed with PBS, and seeded onto upper chamber (primarily coated with Matrigel) to invade.

Abbreviations: RA, all-trans retinoic acid; DexDA, deoxycholic acid-conjugated dextran; ENI0, empty nanoparticles of DexDA-2, I0 $\mu g / m L$; ENI00, empty nanoparticles of DexDA-2, $100 \mu \mathrm{g} / \mathrm{mL}$; RAI, RA I $\mu \mathrm{g} / \mathrm{mL}$; RA10, RA $10 \mu \mathrm{g} / \mathrm{mL}$; RA20, RA $20 \mu \mathrm{g} / \mathrm{mL}$; RA50, RA $50 \mu \mathrm{g} / \mathrm{mL}$; NPI, RA-incorporated nanoparticles of DexDA-2 (RA dose I $\mu \mathrm{g} / \mathrm{mL}$ ); NPI0, RA-incorporated nanoparticles of DexDA-2 (RA dose $10 \mu \mathrm{g} / \mathrm{mL}$ ); NP20, RA-incorporated nanoparticles of DexDA-2 (RA dose $20 \mu \mathrm{g} / \mathrm{mL}$ ); NP50, RAincorporated nanoparticles of DexDA-2 (RA dose $50 \mu \mathrm{g} / \mathrm{mL}$ ); MMP-2, matrix metalloproteinases-2.

cells. As shown in Figure 7, tumor nodules in mouse lung were generated by pulmonary metastasis of tumor cells. Tumor nodules were decreased by treatment of RA or RAincorporated nanoparticles, as shown in Figure 7. In particular, RA-incorporated nanoparticles markedly inhibited generation of tumor nodule in the mouse lung, indicating that RA-incorporated nanoparticles have sustained release properties and are favorable in longer blood-circulation time of drug. These properties might result in better antiinvasive and antimetastatic activity of nanoparticles than the drug itself.

\section{Discussion}

The self-aggregation properties of amphiphilic macromolecules are regarded as a driving force of nanoparticle formation. ${ }^{1,5-7}$ Nanoaggregates or polymeric micelles are appropriate vehicles for incorporation of hydrophobic drugs, due to their self-association characteristics and hydrophobic interaction in the aqueous environment. In particular, small nanoaggregates or nanoparticles have such advantages as long blood circulation of drugs, avoiding unwanted side effects of anticancer drugs, ease of administration, and solubilization of hydrophobic drugs. We chose RA as an anticancer agent 


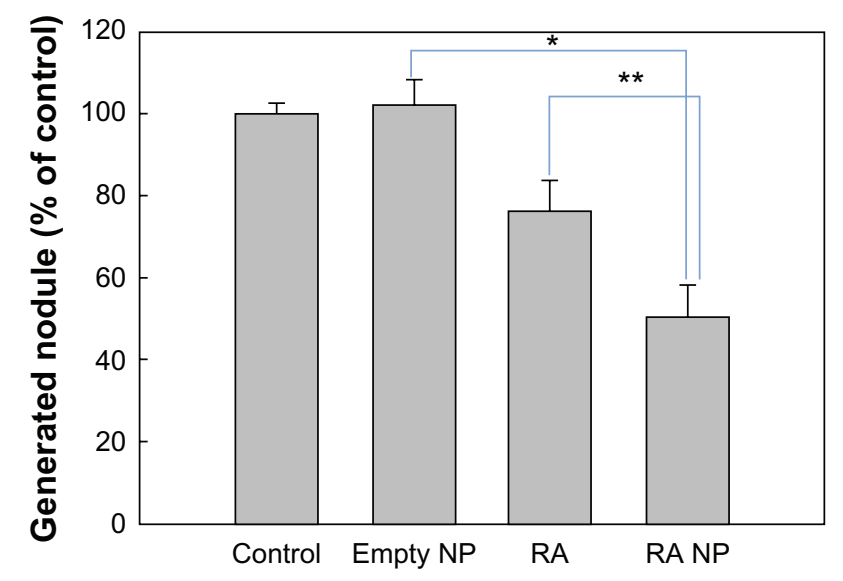

Figure 7 The effect of RA-incorporated nanoparticles of DexDA-2 on the pulmonary metastasis of CT26 cells in vivo.

Notes: $* P<0.001$; $* * P<0.01$.

Abbreviations: RA, all-trans retinoic acid; NP, nanoparticles.

because it is believed to be safe for the human body and it has antitumor activity, ie, suppression of tumor cell proliferation and inhibition of tumor cell invasion. ${ }^{8-14}$ However, rapid clearance from the blood circulation and poor aqueous solubility of RA limits clinical application. ${ }^{18}$ From these points of view, nanoparticles formed by a self-aggregation process can solve these problems. ${ }^{5-7,20,21,24}$ Nanoparticles or polymeric micelles are known to be effective in extending blood circulation times of anticancer drugs, decreasing side effects of drugs, and suppression of tumor cell growth. ${ }^{3,6,24}$ As shown in Figure 1, Figure 2, and Table 1, we prepared RA-incorporated DexDA nanoparticles for antitumor drug delivery. RA was incorporated into the DexDA nanoparticles through hydrophobic interaction with hydrophobic moiety, DA, in the nanoparticles. Liphophilic drug at higher drug contents is known to aggregate or crystallize in the core of the nanoparticles, and then crystallized drug releases more slowly than that in lower drug loading. ${ }^{3}$ As shown in Figure 3, RA with higher drug content was released slowly from the nanoparticles compared to lower drug loading, indicating that RA might be crystallized in the core of the nanoparticles and released slowly. In a previous report by Jeong et al, ${ }^{27} \mathrm{RA}$ was crystallized in the core of the nanoparticles at higher drug loading, and solubilization of crystallized drug into the release medium was delayed compared to molecular dispersion of drug. They demonstrated that RA can be crystallized in the nanoparticles at higher drug loading through analysis of powder X-ray diffraction. The particle size of DexDA nanoparticles was increased by drug loading, and this increased size might induce a slower drug release at higher drug contents, as shown in Figures 2 and 3.
Since RA is known to have antiproliferative and antiinvasive activity against tumor cells, RA-incorporated nanoparticles were assessed by MTT proliferation test, gelatin zymography, and Matrigel invasion assay. Prior to investigation of antitumor activity, the intrinsic toxicity of empty nanoparticles of DexDA-2 was checked using RAW264.7 mouse macrophage cells. As shown in Figure 4, empty nanoparticles of DexDA did not significantly affect the viability of normal cells, ie, higher than $80 \%$ (versus control) of cells survived at higher than $100 \mu \mathrm{g} / \mathrm{mL}$ of DexDA conjugates. These results indicated that DexDA conjugates do not have acute toxicity and are compatible with normal cells. Figure 5 shows the antiproliferative activity of RA and RAincorporated nanoparticles. RA-incorporated DexDA nanoparticles inhibit proliferation of tumor cells. Furthermore, we suggest that the reason for the differences in cell proliferation between RA and nanoparticles was the sustained-release properties of RA-incorporated nanoparticles.

Since RA is known to inhibit local invasion and/or metastasis of tumor cells, ${ }^{13}$ anti-invasive and antimetastatic activity of RA and RA-incorporated nanoparticles were investigated using Matrigel invasion assay, gelatin zymography, and an in vivo pulmonary metastasis model of CT26 cells. As shown in Figure 6A, RA or RA-incorporated nanoparticles dose-dependently inhibited invasion of tumor cells on Matrigel invasion assay in vitro. Antimetastatic effects of RA against mouse pulmonary metastatic model have been investigated by several researchers. ${ }^{25,27}$ We also demonstrated the antimetastatic activity of RA-incorporated nanoparticles using mouse pulmonary metastatic model of CT26 cells, as shown in Figure 7. Suzuki et al reported that RA-encapsulated cationic liposome suppressed pulmonary metastasis of tumor cells. ${ }^{26}$ Siddikuzzaman and Grace also reported that RA-incorporated liposome was more effective in inhibiting metastatic lung cancer than RA. ${ }^{28}$ In our results, RA-incorporated nanoparticles also had higher effectiveness in suppression of metastatic potential of tumor cells than RA itself.

In conclusion, RA-incorporated DexDA nanoparticles were prepared to test their antitumoral activity in in vitro and in vivo conditions. RA-incorporated nanoparticles showed similar antiproliferative and anti-invasive activity against CT26 tumor cells compared to RA itself. However, RA-incorporated DexDA nanoparticles showed higher antimetastatic potential than RA itself. We suggest that RAincorporated DexDA nanoparticles are promising vehicles to inhibit tumor metastasis. 


\section{Acknowledgment}

This work was partially supported by a grant-in-aid (PJ007538) for scientific research from the Ministry for Food, Agriculture, Forestry and Fisheries of Korea. This work was partially supported by the National Agenda Project (NAP), funded by the Korea Research Council of Fundamental Science and Technology (P-09-JC-LU63-C01).

\section{Disclosure}

The authors report no conflicts of interest in this work.

\section{References}

1. Akiyoshi K, Kobayashi S, Shichibe S, et al. Self-assembled hydrogel nanoparticle of cholesterol-bearing pullulan as a carrier of protein drugs: complexation and stabilization of insulin. J Control Release. 1998;54: 313-320.

2. Allemann E, Gurny R, Doelker E. Drug-loaded nanoparticlespreparation methods and drug targeting issues. Eur J Pharm Biopharm. 1993;39:173-191.

3. Gref R, Minamitake Y, Peracchia MT, Trubetskoy V, Torchilin V, Langer R. Biodegradable long-circulating polymeric nanospheres. Science. 1994;263:1600-1603.

4. Kreuter J. Nanoparticle-based drug delivery system. J Control Release. 1991;16:169-176.

5. Jeong YI, Cheon JB, Kim SH, et al. Clonazepam release from core-shell type nanoparticles in vitro. J Control Release. 1998;51:169-178.

6. Yokoyama M, Okano T, Sakurai Y, Ekimoto H, Shibazaki C, Kataoka K. Toxicity and antitumor activity against solid tumors of micelle-forming polymeric anticancer drug and its extremely long circulation in blood. Cancer Res. 1991;51:3229-3236.

7. Jeong YI, Choi KC, Song CE. Doxorubicin release from core-shell type nanoparticles of poly(DL-lactide-co-glycolide)-grafted dextran. Arch Pharm Res. 2006;29:712-719.

8. Bouterfa H, Picht T, Keb D, et al. Retinoids inhibit human glioma cell proliferation and migration in primary cell cultures but not in established cell lines. Neurosurgery. 2000;46:419-430.

9. Giannini F, Maestro R, Vukosavljevic T, Pomponi F, Boiocchi M. All-trans, 13-cis and 9-cis retinoic acids induce a fully reversible growth inhibition in HNSCC cell lines: implications for in vivo retinoic acid use. Int J Cancer. 1997;70:194-200.

10. Huang EJ, Ye YC, Chen SR, et al. Use of all-trans retinoic acid in the treatment of acute promyelocytic leukemia. Blood. 1988;72:567-572.

11. Kalmekerian GP, Jasti RK, Celano P, Nelkin BD, Marby M. All-trans retinoic acid alters myc gene expression and inhibits in vitro progression in small-cell lung cancer. Cell Growth Differ. 1994;5:55-60.

12. Krupitza G, Hulla W, Harant H, et al. Retinoic acid induced death of ovarian carcinoma cells correlates with c-myc stimulation. Int J Cancer. $1995 ; 61: 649-657$.
13. Rotan R. Retinoids as modulators of tumor cell invasion and metastasis. Semin Cancer Biol. 1991;2:197-208.

14. Das A, Banik NL, Ray SK. Retinoids induced astrocytic differentiation with down regulation of telomerase activity and enhanced sensitivity to taxol for apoptosis in human glioblastoma T98G and U87MG cells. J Neurooncol. 2008;87:9-22.

15. Conley BA, Egorin MJ, Sridaha R, et al. Phase I clinical trial of all-trans-retinoic acid with correlation of its pharmacokinetics and pharmacodynamics. Cancer Chem Pharm. 1997;39:291-299.

16. Frankel SR, Eardley A, Lauwers G, Weiss M, Warrell RP Jr. The "retinoic acid syndrome" in acute promyelocytic leukemia. Ann Intern Med. 1992;117:292-296.

17. Muindi JRF, Frankel SR, Miller WH, et al. Continuous treatment with all-trans-retinoic acid causes a progressive reduction in plasma drug concentrations: implications for relapse and retinoid "resistance" in patients with acute promyelocytic leukemia. Blood. 1992;79: 299-303.

18. Szuts EZ, Harosi FI. Solubility of retinoids in water. Arch Biochem Biophys. 1991;287:297-304.

19. Estey E, Thall PF, Mehta K, et al. Alterations in tretinoin pharmacokinetics following administration of liposomal all-trans retinoic acid. Blood. 1996;87:3650-3654.

20. Jeong YI, Kim SH, Jung TY, et al. Polyion complex micelles composed of all-trans retinoic acid and poly(ethylene glycol)-grafted-chitosan. J Pharm Sci. 2006;95:2348-2360.

21. Lim SJ, Kim CK. Formulation parameters determining the physicochemical characteristics of solid lipid nanoparticles loaded with all-trans retinoic acid. Int J Pharm. 2002;243:135-146.

22. Choi Y, Kim SY, Kim SH, Lee KS, Kim C, Byun Y. Long-term delivery of all-trans-retinoic acid using biodegradable PLLA/PEG-PLLA blended microspheres. Int J Pharm. 2001;215:67-81.

23. Ezpeleta I, Irache JM, Stainmesse S, et al. Gliadin nanoparticles for the controlled release of all-trans retinoic acid. Int J Pharm. 1996;131: 191-200.

24. Park KB, Jeong YL, Choi KC, Kim SG, Kim HK. Adriamycinincorporated nanoparticles of deoxycholic acid-conjugated dextran: antitumor activity against CT26 colon carcinoma. J Nanosci Nanotechnol. 2011;11:4240-4249.

25. Charoensit P, Kawakami S, Higuchi Y, Yamashita F, Hashida M. Enhanced growth inhibition of metastatic lung tumors by intravenous injection of ATRA-cationic liposome/IL-12 pDNA complexes in mice. Cancer Gene Ther. 2010;17:512-522.

26. Suzuki S, Kawakami S, Chansri N, Yamashita F, Hashida M. Inhibition of pulmonary metastasis in mice by all-trans retinoic acid incorporated in cationic liposomes. J Control Release. 2006;116:58-63.

27. Jeong YI, Kim DG, Jang MK, Nah JW, Kim YB. All-trans retinoic acid release from surfactant-free nanoparticles of poly(DL-lactide-coglycolide). Macromol Res. 2008;16:717-724.

28. Siddikuzzaman, Grace VM. Inhibition of metastatic lung cancer in C57BL/6 mice by liposome encapsulated all trans retinoic acid (ATRA). Int Immunopharmacol. 2012;14:570-579.
International Journal of Nanomedicine

\section{Publish your work in this journal}

The International Journal of Nanomedicine is an international, peerreviewed journal focusing on the application of nanotechnology in diagnostics, therapeutics, and drug delivery systems throughout the biomedical field. This journal is indexed on PubMed Central,

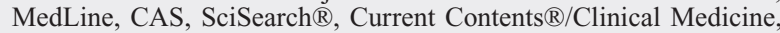

\section{Dovepress}

Journal Citation Reports/Science Edition, EMBase, Scopus and the Elsevier Bibliographic databases. The manuscript management system is completely online and includes a very quick and fair peer-review system, which is all easy to use. Visit http://www.dovepress.com/ testimonials.php to read real quotes from published authors. 